\title{
How to Oxidize Methane with a P450 Enzyme
}

Organo- and Biocatalysis

\section{Key words}

methane oxidation monooxygenases perfluoro carboxylic acids

protein tuning

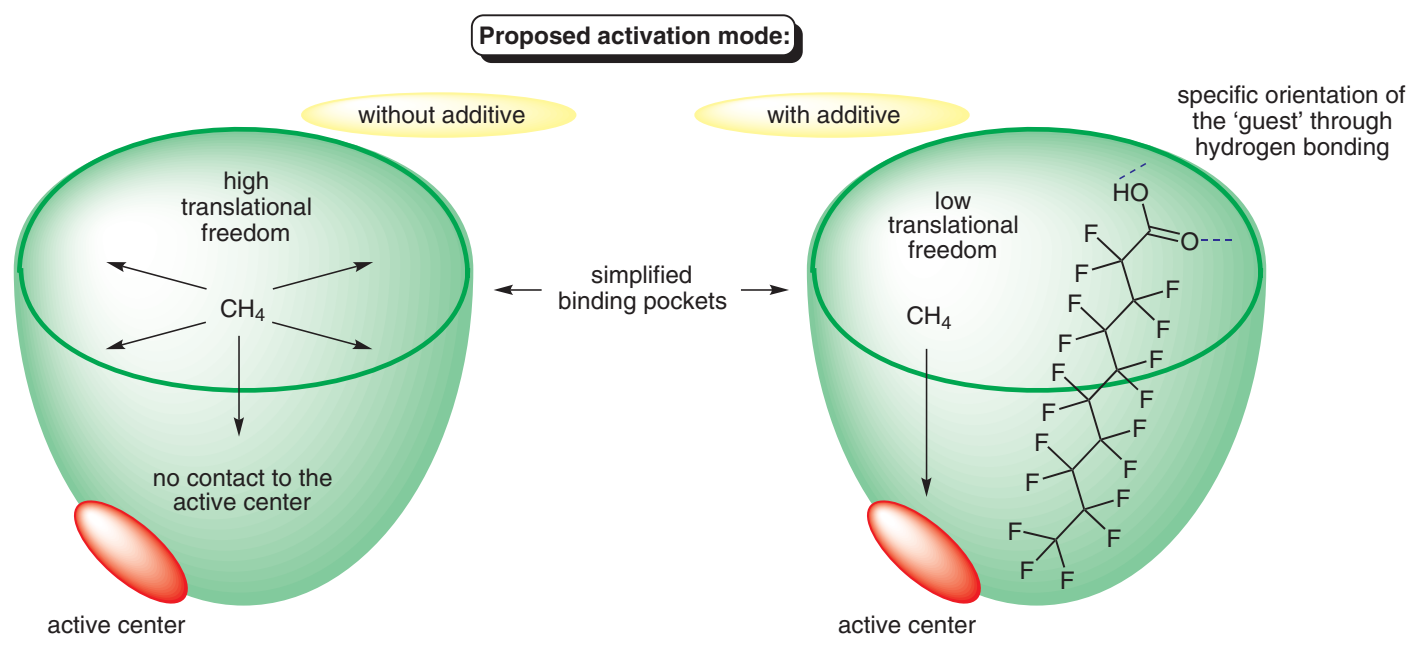

Significance: The enzyme-catalyzed oxidation of simple liquid and gaseous alkanes, among others methane, is reported. The biocatalytic system involved uses a cytochrome P450 BM3 enzyme from Bacillus megaterium, a heme-dependent monooxygenase, in combination with perfluorinated carboxylic acids. The role of this acid additive is to modulate guest-host interactions in the pocket, thus triggering the catalytic activation as well as dramatically decreasing the free space inside the binding pocket. With this lower translational freedom, the approach of the alkane to the active center is facilitated and increases the cataIytic turnover in some cases by several orders of magnitude. The experimental findings were further supported by UV/Vis spectroscopy and molecular dynamics simulations, also providing hints that for different alkane substrates different acid additives might be essential.
Comment: The high potential of biocatalysis is unambiguous since it uses environmentally benign reaction conditions paired with high catalytic turnover numbers. To provide new biocatalysts the most commonly accepted strategies are rational design of enzymes and directed evolution towards more active mutants. Using a merger of these strategies, Rao, Wong and co-workers, among others, were able to identify enzymes capable of oxidizing rather simple alkanes (Angew. Chem. Int. Ed. 2005, 44, 4029). The strategy presented in the current work, however, is much simpler and can be considered as a chemical tuning, avoiding difficult and time-consuming enzyme modifications. Since even the biocatalytic oxidation of methane was realized, which is difficult to obtain otherwise, this strategy will surely find more applications and stimulate further research. 